\title{
Delayed neutron emission near the shell-closures
}

\author{
Ivan Borzov ${ }^{1, a}$ \\ ${ }^{1}$ Bogoliubov Laboratory of Theoretical Physics, JINR, 141980 Dubna, Moscow region, Russia
}

\begin{abstract}
The self-consistent Density Functional + Continuum QRPA approach (DF+CQRPA) provides a good description of the recent experimental beta-decay half-lives and delayed neutron emission branchings for the nuclei approaching to (and beyond) the neutron closed shells $N=28,50,82$. Predictions of beta-decay properties are more reliable than the ones of standard global approaches traditionally used for the $r$-process modelling. An impact of the quasi-particle phonon coupling on the delayed multi-neutron emission rates $P_{2 \mathrm{n}}$, $P_{3 \mathrm{n}, \ldots}$ near the closed shells is also discussed.
\end{abstract}

\section{Introduction}

The $\beta$-decay half-lives and $\beta$-delayed neutron emission probabilities of very neutron-rich nuclei are of great value for nuclear structure theory. Reliable masses and betarates of extremely neutron-rich nuclei are indispensable for the $r$-process modeling. Accurate beta-decay data on the fission products are important for safety studies of advanced nuclear reactors.

A purely integral quantities, $\beta$-decay half-lives and $\beta$ delayed neutron emission probabilities, give an insight to isospin response of nuclei far from the stability. A combined analysis of total $\beta$-decay half-lives and multineutron emission rates ( $P_{\mathrm{xn}}$-values) enables one to reconstruct the beta-strength functions carrying back information on the nuclear density functional at high isospinasymmetry regime. The beta-decay studies show that not only the neutron-halo nuclei near the drip-line reveal the features of weakly bound open quantum systems. Similar effects arise in neutron-rich nuclei because of "shellerosion" induced by proton-neutron tensor interaction.

A substantial amount of more precise $\beta$-decay data on the fission and fragmentation products are expected to come from the acting radioactive beam facilities: ISOLDE-CERN, ALTO, RIKEN, TRIUMF, NSCL, as well as constructed FAIR, Spiral-2, HIE-CERN facilities. An importance of this field has been stated in the IAEA Project of creating new data base of the beta-decay and delayed neutron emission rates [1]. It will acquire accurate experimental data and theoretical predictions for nuclides beyond the reach of existing or planned facilities. In this respect, the fully microscopic models are of a special importance, as they ensure more reliable extrapolation of nuclear data to extreme $N / Z$ ratios.

Based on the self-consistent description of the ground state properties within the local energy-density functional theory [2], the approach to the large-scale continuum

\footnotetext{
ae-mail: ibor48@mail.ru
}

quasi-particle random phase approximation (QRPA) calculations of the allowed Gamow-Teller (GT) and firstforbidden (FF) beta decays has been developed in [3]. In the report, the ground state properties and half-lives as well as delayed neutron-emission rates of (near) spherical nuclides near the neutron closed shells $N=28,50,82$ is presented and compared with the recent experimental data [4-7]. Comparison with the standard global Finite Range Droplet Model (FRDM) [8] used for the astrophysical modeling is also be done. An emphasis is made on the constraints imposed by the half-lives as well as delayed neutron-emission rates on the beta-decay strength functions. A possible impact of the quasi-particle phonon coupling $[9,10]$ on the delayed multi-neutron emission rates $P_{2 \mathrm{n}}, P_{3 \mathrm{n}, \ldots}$ near the closed shells is discussed as well.

\section{Theoretical background}

The self-consistent model for the GT and first-forbidden decays has been developed in [3] in which a detailed description and comparison with existing semi-microscopic global models can be found. The DF+CQRPA model treats the ground state and $\beta$-decay properties of (quasi)spherical nuclei.

1. The ground state properties are derived self-consistently in the energy-density functional theory. The present framework also exploits a new functional DF3a [11] and has a provision to fix (before variation) the ground state spin-parity of the parent (daughter) isobaric companion [4].

2. For excited states we follow an approximate treatment [3] in which the scalar and spin-isospin components of the DF can be approximately decoupled. This allows for independent nucleon-nucleon (NN) interactions in the scalar and spin-isospin channels. The strength parameters are considered as universal ( $A$-independent) constants.

3 . The $\beta$-strength functions are calculated within the continuum QRPA of the finite Fermi system theory. For 
the spin-isospin effective $\mathrm{NN}$-interaction in the $\mathrm{p}$-h channel a finite-range $\delta+\pi+\rho$ form is assumed. The nuclear medium modified one- $\pi$ and one- $\rho$ exchange terms, are important for the spin-isospin responses [3].

4. The correlations beyond the QRPA are included by re-scaling the spin-dependent multipole operators by the same energy-independent quenching factor $Q^{1 / 2}=$ $\left(g_{A} / G_{A}\right)$. The one-pion component of the residual interaction is quenched by the same factor $Q$.

5 . For the calculation of the $\beta$-decay half-lives we have considered allowed and first-forbidden transitions treated in terms of the reduced multipole operators depending on the space and spin variables [3].

6. The DF+CQRPA framework was successfully used for calculations of the beta-decay half-lives [3] and magnetic moments [12].

7. For a simple estimate of an impact of the $n p-$ $n h$ configurations on the $\beta$-strength functions, we use a spreading width of excitations $\Gamma \downarrow \approx \alpha \omega$ as in [13]. Also we studied an influence of quasiparticle-phonon coupling included via finite rank separable approximation (FRSA) [9, $10]$.

\section{Results}

Below the performance of the DF+CQRPA approach is exemplified. The main features of the $\beta$-decay and multineutron emission rates are described for the isotopes near the magic shell-closures in the neutron-rich sector.

\section{1 ${ }^{48}$ Ca region}

The region of $Z \sim 0, N>28$ is of great interest in the view of new experimental possibilities opened at FLNR, JINR for exploiting the $\mathrm{Ca}$ beams. In Fig. 1, the integral betadecay characteristics calculated from the DF3+CQRPA beta-strength functions are compared with the recent data for the potassium isotopes. The experimental $\beta$-decay half-lives and total $P_{\mathrm{n}}$ values are reasonably well described by our model.The experimentally observed drop of the $P_{\mathrm{n}}$ value at $A=50$ is related to occurrence of the additional first-forbidden transitions mediated by the odd-particles in the course of filling of the $N=32$ and 34 subshells. Further steady increase of the $P_{\mathrm{n}}$ value is due to the impact of strong GT decays built on the $v 1 f_{7 / 2}-\pi 1 f_{7 / 2}$ transitions.

\subsection{Nuclei below $Z=28$ shell}

The Co isotopes with $N \leqslant 50$ are a clean case for which the beta-decay characteristics are defined by the GamowTeller strength function. In Fig. 2, we show the GT strength distribution for ${ }^{74} \mathrm{Co}$ (in terms of $\log (\mathrm{ft})$ values). Importantly, the resulting $T_{1 / 2}$ and $P_{\mathrm{n}}$ values are very sensitive to the GT strength near the one-neutron separation energy.

As seen from Fig. 3, our calculations underestimate the half-lives and overestimate a bit the $P_{\mathrm{n}}$ values. In general, a very regular A-dependence of the calculated beta-decay observables agrees with the data and confirms a predominantly Gamow-Teller character of the beta-decay in this isotopic chain.
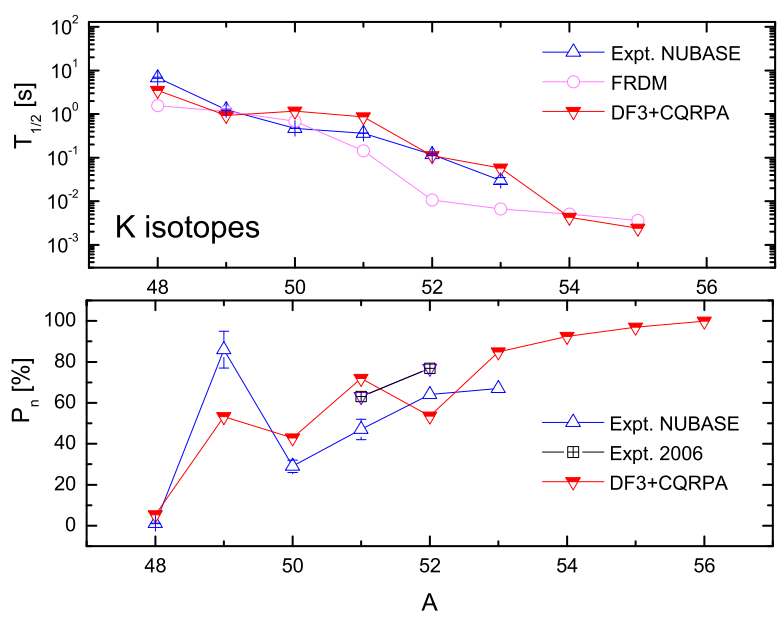

Figure 1. (Color online) Beta-decay half-lives and total $P_{\mathrm{n}}$ values for the $\mathrm{K}$ (potassium) isotopic chain. The experimental data have been obtained from the NUBASE evaluation [14]. The data of Ref. [15] are also shown.

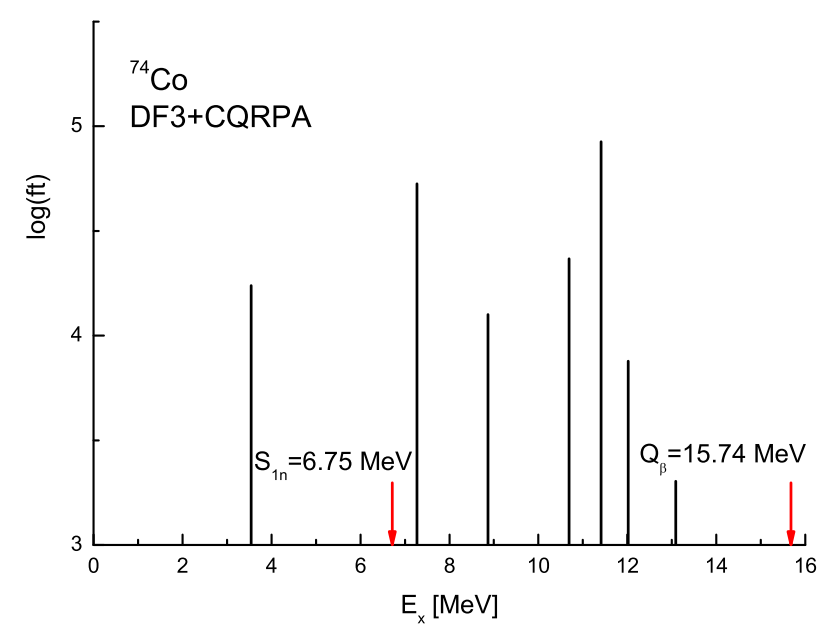

Figure 2. (Color online) The Gamow-Teller strength function for the decay of the ${ }^{74} \mathrm{Co}$ parent nucleus.

\section{$3.3{ }^{78} \mathrm{Ni}$ region}

The most neutron-rich region of the nuclear chart in vicinity of ${ }^{78} \mathrm{Ni}$ may serve as a laboratory for exploring nuclear structure under extreme conditions. The $Z \sim 28$ isotopes beyond $N=50$ may be affected by weakened neutron binding due to the ground-state spin inversion and possible existence of new neutron shell closures $N=56-58$. An existence of neutron-halo structures is not excluded revealing unusual features of weakly bound open quantum system. These structural changes naturally impact the betastrength function.

An evidence for new spherical subshell closure at $N=58$ was experimentally obtained recently for ${ }^{83-86} \mathrm{Ga}$ isotopes $[4,5]$. This subshell formed of two nearly degenerated orbitals may originate from migration of the neutron $2 d_{5 / 2}$ and $3 s_{1 / 2}$ orbitals causing a reduction of $N=50$ shell gap and some weakening of the ${ }^{78} \mathrm{Ni}$ doubly-magic core. Another peculiarity of this region is that the neutron- 

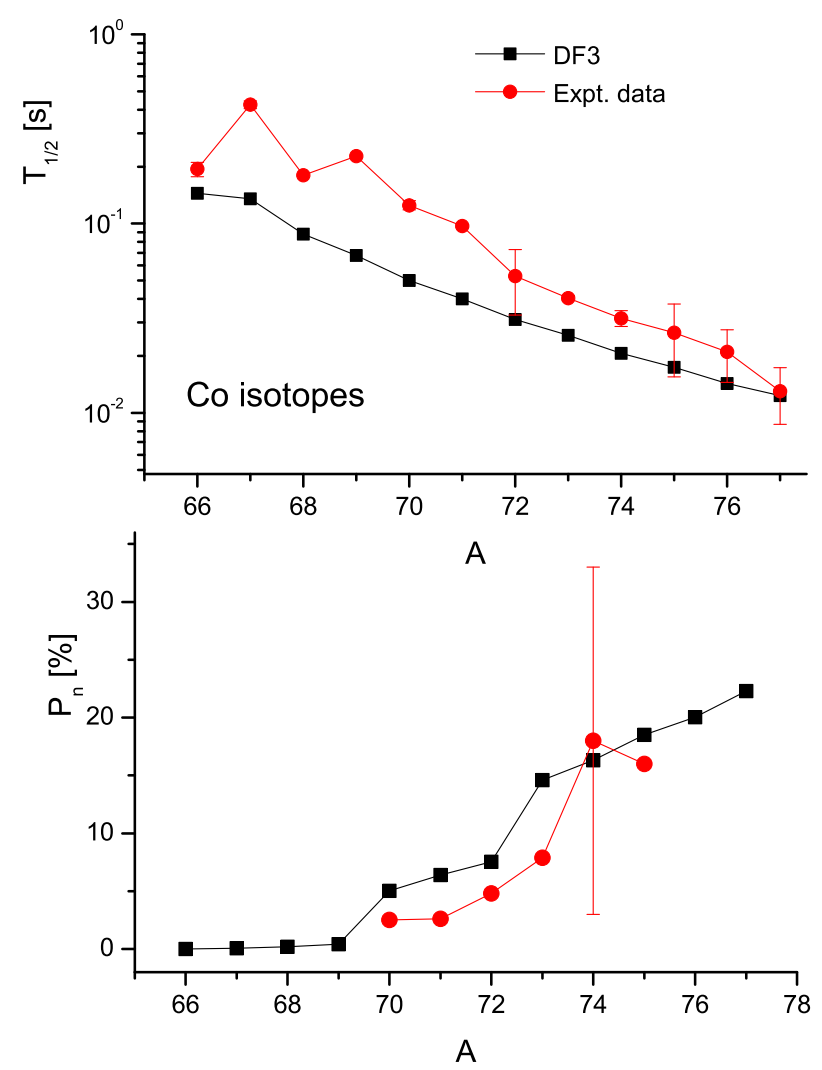

Figure 3. (Color online) The $\beta$-decay half-lives and total $P_{\mathrm{n}}$ values for the Co isotopic chain. Experimental data are taken from Refs. [6, 14].

proton tensor interaction triggers a crossing of the proton $2 p_{3 / 2}$ and $1 f_{5 / 2}$ levels for nuclei beyond $Z=28$. Resulting ground-state spin inversion has well been confirmed in magnetic moment measurements for $\mathrm{Cu}$ isotopes (see Ref. [12]).

Studying the $\beta$-decay of very neutron-rich nuclides with $N>50$ in vicinity of the ${ }^{78} \mathrm{Ni}$ one meets with another difficulty. After crossing the closed neutron shells the neutrons fill positive parity orbitals while protons occupy negative parity orbitals and the first-forbidden transitions become possible. The share of the FF decays to the total halflife increases substantially for isotopes beyond the $N=50$ shell. Clearly, in the scheme including both the GT and FF decays, the total half-lives for Ga chain at $N>50$ depend on the ordering of the orbitals in the neutron $(N=58)$ as well as proton subshells. Additional measurements of the delayed multi-neutron probabilities would have provided us with important information on the beta-strength distributions within the sub-spaces corresponding to emission of $n=0,1,2$ etc neutrons.

Below we discuss the case of ${ }^{86} \mathrm{Ga}$ : the most "powerful" $2 n$ emitter in medium heavy nuclei region $\left(P_{2 \mathrm{n}}=\right.$ $20 \pm 10 \%$ [16]). The "DF3a+CQRPA" model is extended to account for spin inversion in the ${ }^{78} \mathrm{Ni}$ region. The approach is consistent with the systematics of the spin-parity of the ground states of $\mathrm{Cu}$ isotopes (understood as driven by the neutron-proton interaction when filling the $v 1 g_{9 / 2}$

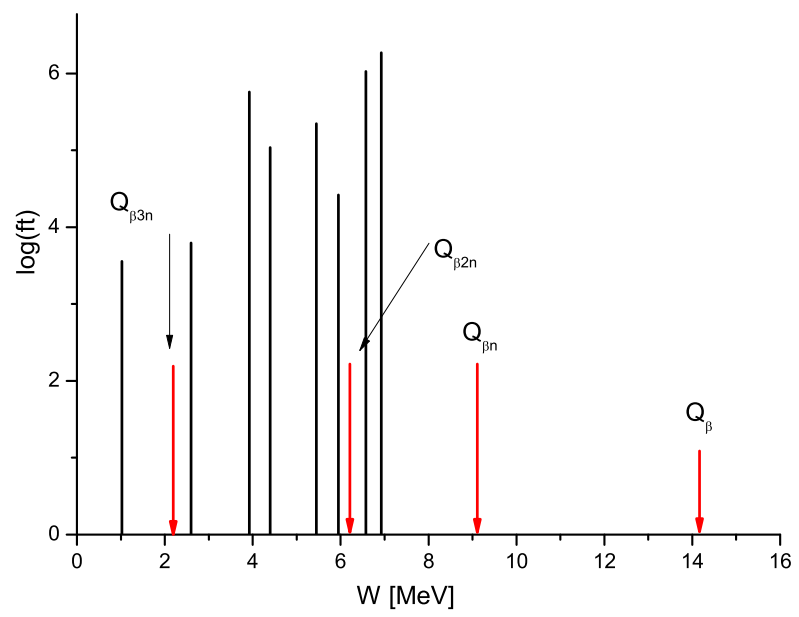

Figure 4. (Color online) The Gamow-Teller $\beta$-decay strength functions for ${ }^{86} \mathrm{Ga}$ plotted as a function of transition energy.

shell [17]). The calculation fixing the ground state to $\pi 1 f_{5 / 2}$ single-particle state reproduces both ${ }^{81-84} \mathrm{Ga}$ and new ${ }^{86} \mathrm{Ga}$ half-lives. No local adjustment was made.

Description of the $P_{\mathrm{xn}}$ values in ${ }^{86} \mathrm{Ga}$ is more difficult. They are mostly determined by the GT strength function, since the FF strengths $(\Delta J=0,1,2)$ being concentrated at the excitation energies below $S_{1 n}$ (transition energies higher the $Q_{\beta n}$ ). As seen from Fig. 4, our CQRPA calculation shows some deficiency of the GT strength within one-neutron emission sub-space. This is due to neglect of the GT strength fragmentation which may be caused by the effects beyond the simple $1 p-1 h$ description and by deformation (both are not included in the DF3a+CQRPA model).

For the $\mathrm{Ga}$ isotope chain we estimated an impact of the $n p-n h$ configurations beyond the CQRPA via spreading width $\Gamma \downarrow \approx \alpha \omega$, as suggested in Ref. [13]. Including the spreading (Figs. 5-7) makes the half-lives shorter and closer to the data [5], total $P_{\mathrm{n}}$ values increase, and the $P_{\text {xn }}$ values are now $P_{1 \mathrm{n}}=28 \%>P_{2 \mathrm{n}}=22 \%$ [16]. This is a strong argument in favour of accounting for a quasiparticle-phonon coupling.

\section{$3.4{ }^{132} \mathrm{Sn}$ region}

In ${ }^{132} \mathrm{Sn}$ region the GT and FF decays contribute differently to the beta-decay rates for the $Z<50$ and $Z>50$ isotopes. The intensive GT decays in the $Z<50 \mathrm{nu}-$ clei mostly correspond to the $\left(v 1 g_{7 / 2}, \pi 1 g_{9 / 2}\right)$ configuration. The high-energy GT decays contribute strongly to the total half-lives of the nuclei with $Z<50, N<82$. Then at $N>82$, the high-energy first-forbidden decays become opened which are mainly related to the $\left(v 1 h_{11 / 2}\right.$, $\pi 1 g_{9 / 2}$ ) configuration. In Fig. 8, we exemplify the above said for the Pd isotopic chain. Importantly, the $1 p-1 h$ DF3+CQRPA calculations [18] slightly overestimate the recent RIKEN data [7]. At the same time our FRSA calculation [10] including both the tensor interaction and $2 p-2 h$ configuration effects shows a very good agreement for ${ }^{128} \mathrm{Pd}$. 


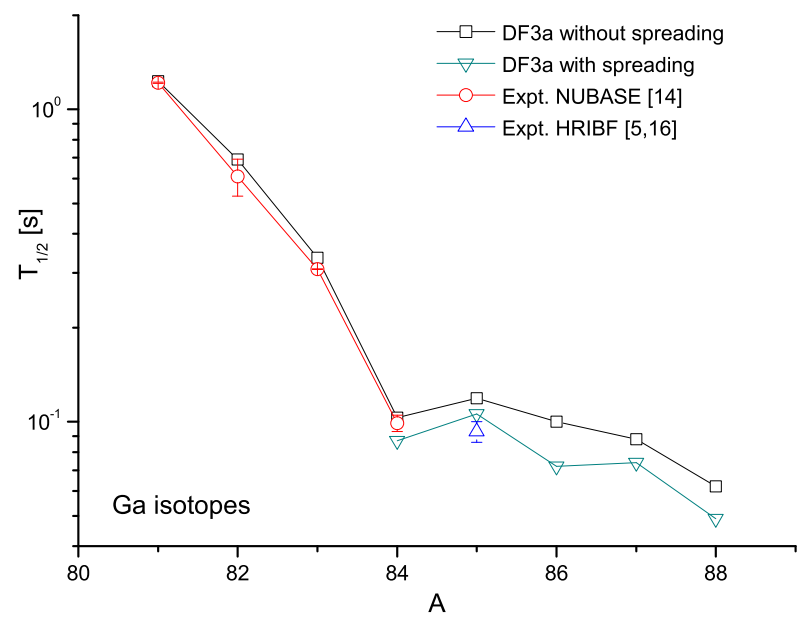

Figure 5. (Color online) The $\beta$-decay half-lives for the Ga isotopic chain calculated without and with inclusion of the spreading width. The experimental data are taken from Refs. [5, 14, 16].

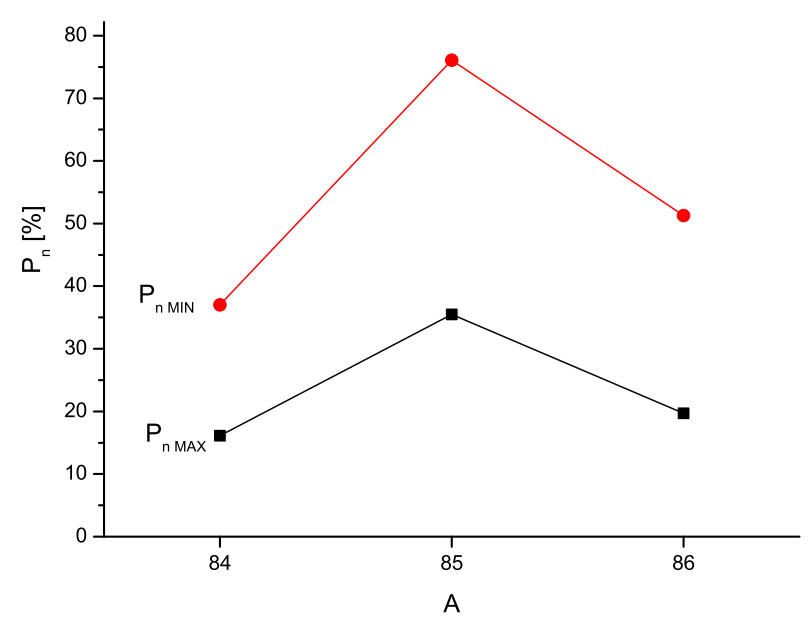

Figure 6. (Color online) The total delayed neutron emission probabilities for the $\mathrm{Ga}$ isotopic chain calculated without $\left(P_{\mathrm{nMIN}}\right)$ and with inclusion of the spreading width $\left(P_{\mathrm{nMAX}}\right)$.

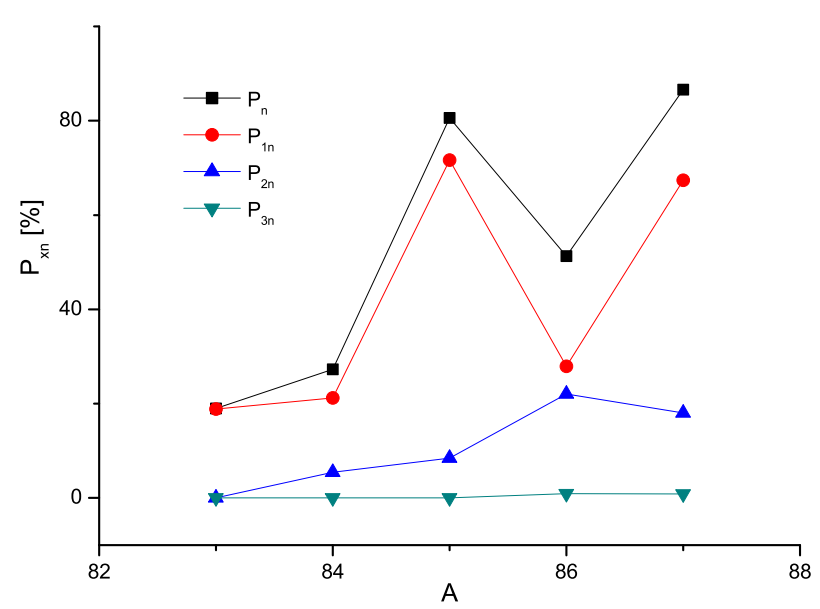

Figure 7. (Color online) The $P_{1 \mathrm{n}, 2 \mathrm{n}, 3 \mathrm{n}}$ and total $P_{\mathrm{n}}$ emission probabilities for the $\mathrm{Ga}$ isotopic chain calculated with inclusion of the spreading width.

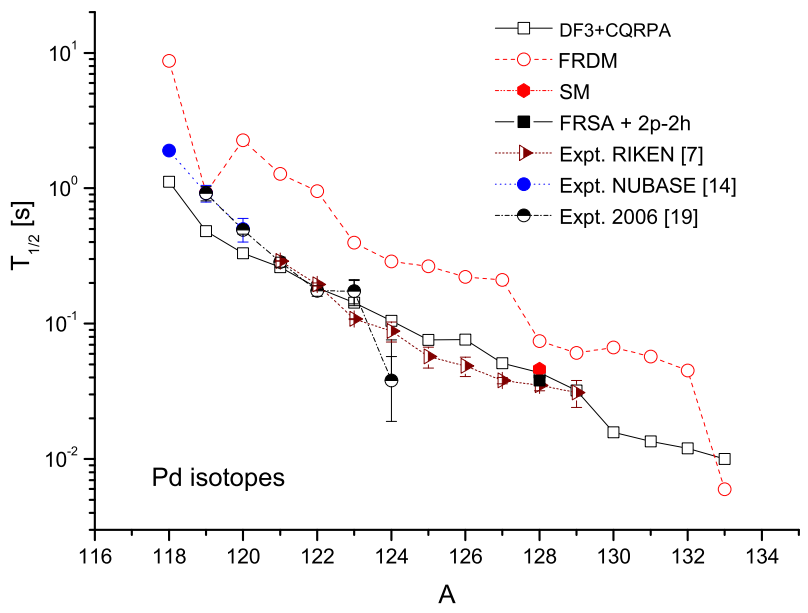

Figure 8. (Color online) Beta-decay half-lives for the Pd isotopic chain. These results are compared to the data $[7,14,19]$ and halflives based on the FRDM [8], the FRSA model including both the tensor interaction and $2 p-2 h$ configuration effects [10], the shell model (SM) [20].

As for nuclei beyond $Z=50$ the $\pi 1 g_{9 / 2}$ orbital is fully occupied, the GT transitions are related to low-energy $\left(v 1 g_{7 / 2}, \pi 1 g_{7 / 2}\right)$ configuration. Due to the phase space effect, the higher energy forbidden transitions which are opened at $N>82$ and related to the $\left(v 2 f_{7 / 2}, \pi 1 g_{7 / 2}\right)$, $\left(v 1 f_{7 / 2}, \pi 2 d_{5 / 2}\right)$ configurations dominate the total halflives. Though, for nuclei with $Z>52$ and $N>82, \pi 1 g_{9 / 2}$ orbital is partially de-blocked due to pairing correlations, the GT transition strength is quenched by the occupancy factor of the proton level $\left(1-v_{\pi 1 g_{9 / 2}}^{2}\right)$.

Probably, the most striking example of half-life reduction due to high-energy forbidden transitions is the kink at ${ }^{134} \mathrm{Sb}(N>82)$ in Fig. 9. Notice, that our calculated half-lives are in rather good agreement with the data. For instance, in ${ }^{136} \mathrm{Sb}$ the $T_{1 / 2}=0.80 \mathrm{~s}$ which is rather close to the experimental value $T_{1 / 2}=0.923 \pm 0.014 \mathrm{~s}$, as the FRDM [8] gives a factor 5 longer half-life.

In Fig. 10, we display the $Q_{\beta}$ values for the Sb isotopes beta-decay and neutron emission thresholds in the daughter nuclei $\left(S_{\mathrm{xn}}\right)$ calculated with the DF3 functional. One may conclude that two-neutron emission for $A>135$ and three neutron emission for $A>138$ are possible. However, compared to $Z<50$ isotopes, the delayed neutron emission branching should be rather suppressed in the Sb isotopes due to the fact that the dominating FF transitions undergo outside the $Q_{\beta \mathrm{n}}$ window. Another complication is that the $P_{\mathrm{xn}}$ values are extremely sensitive to the $S_{\mathrm{xn}}$ values. Possible redistribution of the beta-strengths near the multi-neutron emission thresholds due to quasiparticlephonon coupling is that counts. As seen from Fig. 11, in the ${ }^{136} \mathrm{Sb}$ isotope increasing the $Q_{\beta \mathrm{n}}$ value or decreasing the $Q_{\beta 2 \text { n }}$ thresholds by about $0.5 \mathrm{MeV}$ would have changed the $P_{1 \mathrm{n}}$ and $P_{2 \mathrm{n}}$ values considerably. One has to mention that the accuracy of our calculation of the $Q_{\beta \mathrm{xn}}$ values is about $0.5-1 \mathrm{MeV}$. For instance, in ${ }^{136} \mathrm{Sb}$ our calculations with different versions of the density functional give the total $P_{\mathrm{n}}$ value from $5.4 \%$ to $15.6 \%$ and $P_{2 \mathrm{n}}$ value 


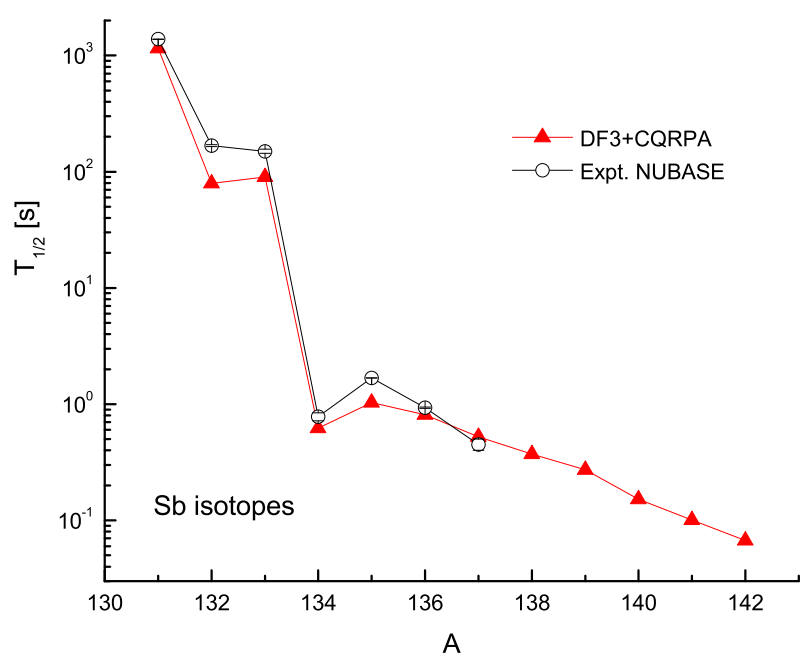

Figure 9. (Color online) Beta-decay half-lives for the Sb isotopic chain. The experimental half-lives were obtained from the NUBASE evaluation [14].

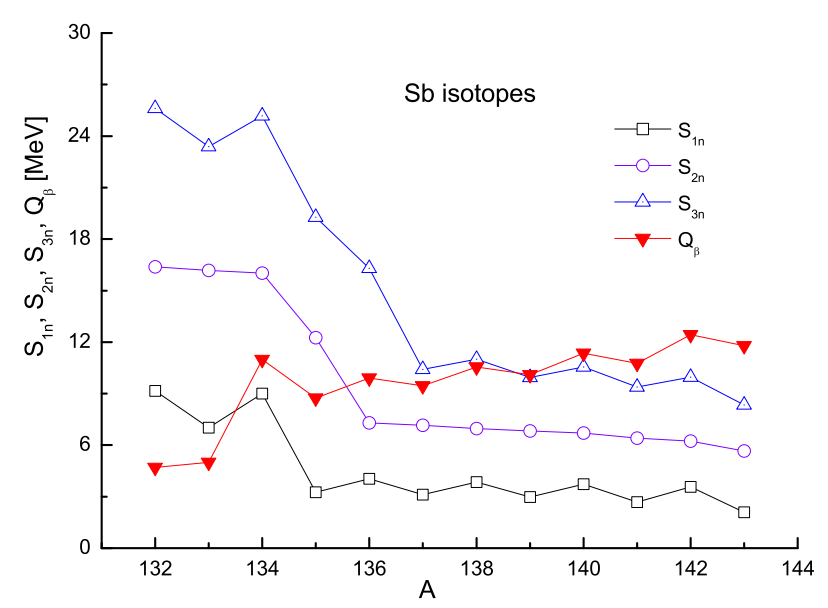

Figure 10. (Color online) $Q_{\beta}$ values and the neutron emission thresholds in daughter nuclei for the $\mathrm{Sb}$ isotopes beta-decay.

from $3.1 \%$ to $5.2 \%$. The experimental values [14] are: $\mathrm{P}_{\mathrm{n}}=16.3 \pm 0.32 \%$ and $P_{2 \mathrm{n}}=0.26 \pm 0.02 \%$. In the photofission measurements at JINR, Dubna [21] it was reported $P_{2 \mathrm{n}}=2.0 \pm 0.2 \%$.

\section{Summary}

With the universal set of the $\mathrm{NN}$-interaction parameters the self-consistent approach to nuclear beta-decay [3] reliably describes both ground-state properties and small amplitude nuclear spin dynamics of (quasi)spherical nuclei in a wide region of the nuclear chart. Microscopic treatment of the GT and FF beta-decays within the single DF+CQRPA framework makes it possible to explain a number of peculiar effects observed in the beta-decay and delayed neutron emission of very neutron-rich nuclei beyond the closed neutron shells. Further extension of the self-consistent DF+CQRPA is related to description of deformed nuclei [22]. Due to a deficiency in extrapolating

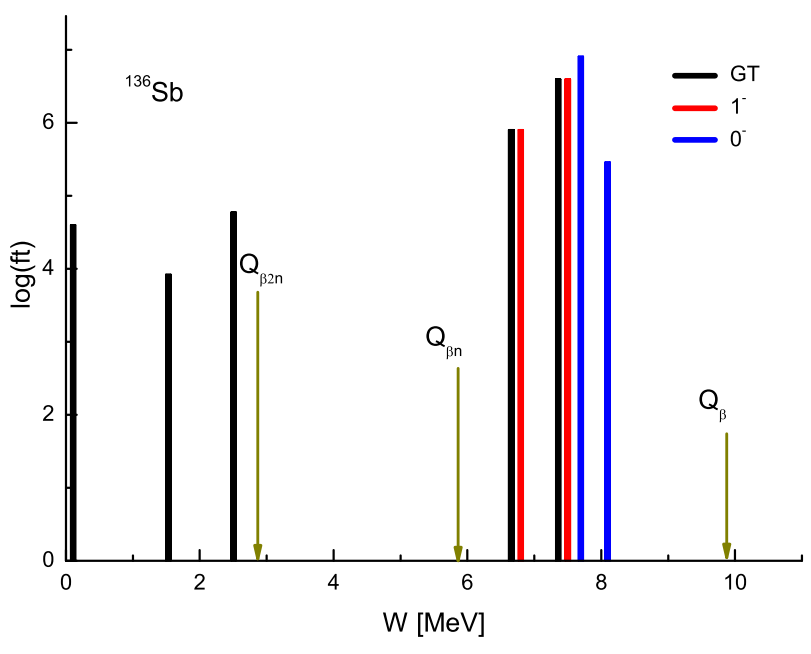

Figure 11. (Color online) The strength functions for the GT and FF decays in ${ }^{136} \mathrm{Sb}$ (plotted in terms of the transition energy)

the model parameters (defined near the line of stability), predictions of semi-empirical and schematic global models should always be taken with some reservation for unstable isotopes near the shell-closures.

The search for delayed multi-neutron emission at JINR, GSI, ALTO, HRIBF and RIKEN would be promising in the region beyond ${ }^{48} \mathrm{Ca}(\mathrm{K}, \mathrm{Ca}, \mathrm{Sc}$ isotopes with $N>30$ ). It is expected to be more favorable (though more difficult) for $Z<50$ (Ag, Cd, In isotopes) than for $Z \geqslant 50$ ( $\mathrm{Sn}, \mathrm{Sb}, \mathrm{Te}$ isotopes). The dedicated $(\beta-n)$ and $(\beta-n-\gamma)$ experiments at JINR, GSI, HRIBF, ALTO, RIKEN using fragmentation reactions and post-accelerated beams at FRIB facilities are indispensable for testing the nuclear structure models far off stability.

\section{References}

[1] D. Abriola et al., IAEA Report, INDC(NDS)-0599 (IAEA, Vienna, 2011)

[2] S.A. Fayans et al., Nucl. Phys. A 676, 49 (2000)

[3] I.N. Borzov, Phys. Rev. C 67, 025802 (2003)

[4] J. Winger et al., Phys. Rev. Lett. 102, 142502 (2009)

[5] M. Madurga et al., Phys. Rev. Lett. 109, 112501 (2012)

[6] Z.Y. Xu et al., Phys. Rev. Lett. 113, 032505 (2014)

[7] G. Lorusso et al., Phys. Rev. Lett. 114,192501 (2015)

[8] P. Möller et al., Phys. Rev. C 67, 055802 (2003)

[9] A.P. Severyukhin et al., Phys. Rev. C 90, 044320 (2014)

[10] A.P. Severyukhin et al., J. Phys.: Conf. Ser. 580, 012051 (2015)

[11] S.V. Tolokonnikov and E.E. Saperstein, Phys. At. Nucl. 73, 1684 (2010)

[12] I.N. Borzov et al., Phys. At. Nucl. 71, 469 (2008)

[13] P.F. Bortignon et al., Nucl. Phys. A 460, 149 (1986)

[14] G. Audi et al., Chin. Phys. C 36, 1157 (2012)

[15] F. Perrot et al., Phys. Rev. C 74, 014313 (2006)

[16] K. Mernik et al., Phys. Rev. Lett. 111, 132502 (2013) 
[17] J. Winger et al., Phys. Rev. C 81, 044303 (2010)

[18] I.N. Borzov et al., Nucl. Phys. A 814, 159 (2008)

[19] F. Montes et al., Phys. Rev. C 73, 035801 (2006)

[20] J.J. Cuenca-García et al., Eur. Phys. J. A 34, 99 (2007)
[21] Yu.P. Gangrsky et al., JINR preprint, P3-2004-125, JINR, Dubna, 2004.

[22] S.V. Tolokonnikov et al., J. Phys. G: Nucl. Part. Phys. 42, 075102 (2015) 